\title{
An Improvement to a Class of Intensity based Spatial Domain Corner Detection Algorithms using Image Fission and Fusion
}

\author{
Ambar Dutta
}

\begin{abstract}
Corners of an object are important as features for the representation and analysis of its shape in computer vision. Corner detection, particularly in real scenes, is still a challenge. Most of the corner detectors found in the literature generate a number of false corners, which is not acceptable in real-life applications. In this paper, an improvement to a class of corner detection algorithms is presented using image fission/fusion. In this approach, a grayscale image is first divided into several bit-planes. A corner detector is applied on all the bit-planes simultaneously and a threshold (bitplane) is obtained using the concept of information gain. Finally, all the higher bit-plane corners are recombined (up to some thresholded bit-plane) to obtain the final set of corners. Here the corner detection algorithm is considered as a binary classification problem. Experimental results show that this improved approach reduces the number of erroneous corner detection relative to existing spatial domain corner detection algorithms. The improvements are established with the help of a number of performance measures proposed by various researchers. The proposed approach works better with respect to computational time also. This approach can easily be utilized in different low-level image processing applications.
\end{abstract}

Keywords: Corner detection, bit plane decomposition, information gain, threshold, non-maximum suppression, performance measure.

\section{INTRODUCTION}

Image corner detection is crucial in various tasks in computer vision which include image registration, object recognition, image matching, structure from motion analysis, scene analysis, and many others. A corner in an image shows a two-dimensional intensity change which makes it distinguishable from its adjacent pixels. In the literature, there exist a considerable number of algorithms to detect corners from digital images suggested by vision researchers. These algorithms are classified into two main groups: intensity-based and boundary-based [1][2]. The first group of methods estimates some cornerness measure directly from the image gray values, whereas the second group first finds the image boundaries and then calculates the maximum

Revised Manuscript Received on December 30, 2019.

* Correspondence Author

Ambar Dutta, Amity Institute of Information Technology, Amity University, Kolkata, India. Email: adutta@kol.amity.edu

(c) The Authors. Published by Blue Eyes Intelligence Engineering and Sciences Publication (BEIESP). This is an open access article under the CC BY-NC-ND license (http://creativecommons.org/licenses/by-nc-nd/4.0/) curvature or inflection points along those boundaries Different researchers provided excellent literature surveys on corner detection algorithms [1] - [6. Here, an improvement to corner detection using image fission/fusion (bit-plane decomposition and recomposition) is proposed, which improves performance of existing corner detection algorithms. Several researchers utilized corner detection algorithms in different applications [7] - [9].The motivation of the present work is as follows. Even better and popular corner detectors fail to detect proper corners in the presence of noise and low contrast images [10]. In this present improvement, which may be applied to any corner detectors, this problem can be resolved. Moreover, application of bit-plane decomposition is a new approach in the area of corner detection. Possibly, this approach has not yet used in corner detection.

The structure of remainder of this paper is as follows. Section 2 includes a short review of popular corner detectors and discusses different performance measures of corner detection algorithms. Section 3 deals with computation of information gain across different bit-planes. An improved approach to corner detection using image fission/fusion is discussed in section 4. Section 5 provides experimental results which prove the superiority of the proposed approach over the original algorithms are presented. Finally, section 6 provides the conclusion of the entire work.

\section{RELATED STUDIES}

\section{A. Existing Algorithms}

Moravec [11] proposed his idea of "points of interest" for corner detection from digital images. Since then researchers in this field have proposed a significant number of corner detectors. In this section, a short review of some of them is presented.

\section{- Intensity-based Corner Detectors}

Kitchen and Rosenfeld [12] applied differential operators containing first and second order partial derivatives of images to extract image corners. The detector is very sensitive to noise [2]. Harris and Stephens [13] extended the Moravec operator by estimating the local autocorrelation using first-order derivatives. The algorithm is computationally very expensive [1]. Smith and Brady [14] proposed a corner detector (popularly known as SUSAN) where they associated with each image point a local area of similar brightness. 
In 1998, a multigrid approach was applied by Trajkovic and Hedley [15] to increase the quality of the detected corners and to reduce the computational complexity. Zheng et al. [6] used the concept of Plessey detector [13] to propose a corner detector using a measure of gradient module of image gradient direction. Bae et al. [16] used two oriented cross operators, COP to present a corner detector. Mikolajczyk and Schmid [17] gave two methods to detect corners which are scaling and affine transformations invariant. Alkaabi and Deravi [18] gave an idea of a corner detector on the basis of pruning candidate corners. The algorithm is computationally efficient. Rosten and Drummond [19] used machine learning based approach to detect high quality corners in images. Dutta et al. [20] applied tri-modal histogram for window-based corner detection. From each of these algorithms explained in this subsection, it is observed that intensity based corner detectors are heavily dependent on a threshold of the cornerness measure. Zeng et al. [21] combined Harris and SUSAN corner detector to propose a more accurate corner detector. Liu et al. [22] improved Harris corner detector, on the basis of second difference, termed as sHarris, to detect text corners for the extraction video caption region. Dutta [23] proposed a corner detector using local information. The author first divided the image into a number of blocks and instead of detecting corners from the entire image, corners are obtained from those blocks which has high information content.

\section{- Boundary-based Corner Detectors}

Davies [24] presented a corner detector using the concept of generalized Hough transform which is robust, accurate and efficient. Ji and Haralick [25] detected corners from chain-encoded digital arcs using a two-step statistical technique which included corner detection and optimization. Mokhtarian and Soumela [26] proposed a curvature scale space (CSS) based approach for corner detection which was operated in two steps - at first Canny edge detector [27] was used for edge detection from the original edge, and then corners are obtained from the edge image at those locations which exhibits a local maximum of absolute curvature. Shen and Wang [28] applied modified Hough transform for corner detection. Guru et al. [29] measured "cornerity index" in order to measure the importance of a corner point to propose a fast and efficient corner detection algorithm. The proposed algorithm is translation, rotation and scaling invariant. Arrebola and Sandoval [30] used the hierarchical computation of a multi-resolution structure to detect corners. Sarfraz et al. [31] applied the change of sign of slope of the curve along the contour to present a simple, robust with respect to noise, accurate and efficient corner detector which works for smooth and irregular planner shapes. Mokhtarian and Mohanna [2] extended curvature scale space corner detector [26], which also worked on multiple scales. The authors also compared the proposed algorithm with some standard corner detectors. Awrangjeb et al. [32] applied the affine-length parameterized curvature to improve curvature scale space corner detector which is robust with respect to affine transformation. Zhang et al. [33] presented a scale multiplication based corner detector which worked in two stages. The first stage consists of the contour extraction from the object of interest which is followed by the computation of the curvature of the contour using Gaussian derivative filters at various scales. Pedrosa and Barcelos [34] detected shape corner points useful for motion tracking and object recognition. Zhang et al. [35] used the determinant of gradient correlation matrices as a cornerness measure of planar curves to detect corners. Shui and Zhang [36] combined the concepts of the boundary-based and intensity-based detection using anisotropic directional derivative representation to extract simple and higher-order corners effectively. They used Canny [27] edge detector for the extraction of image contours.

\section{B. Performance Measures}

For the comparison of the performance of corner detection algorithms, a number of performance measures have been proposed by different researchers. Each of these measures requires exact number of corners present, number of corners detected correctly, number of missed and wrongly detected corners. In this paper, in order to validate the efficiency of the proposed approach, four three performance measures are used which includes detection gradient (DG) [1], detection rate (DR) and error rate (ER) [37], accuracy (ACCU) measure [2] and two other popular measures - precision and recall. For the efficient corner detector, the values of DG, DR, ER and ACCU measures should be as close as 0, 1, 0 and 100 respectively.

\section{INFORMATION GAIN COMPUTATION}

In this section, a brief description on image fission/fusion and information gain computation is presented on which the present improvement is relied upon.

\section{A. Image Fission/Fusion}

The image fission process decomposes the image into a number of bit-planes. A bit-plane may be defined as a set of bits at the same position in the binary representation of a set of decimal numbers as now described. The leftmost (MSB) bit-plane of an image gives the most critical approximation of values in it (in this case). The lower the index number ('grayness') of a bit-plane, the less is its contribution to the process. In fission of a grayscale image containing a maximum of $\mathrm{N}$ gray levels, or indices, the decomposition yields $\log _{2} \mathrm{~N}$ bit-planes, where $\mathrm{N}$ is a power of 2 . If $\mathrm{I}(\mathrm{x}, \mathrm{y})$ denotes the grayvalue of a gray image $\mathrm{I}$ at the pixel $(\mathrm{x}, \mathrm{y})$, where $0 \leq \mathrm{I}(\mathrm{x}, \mathrm{y}) \leq \mathrm{N}-1$, then

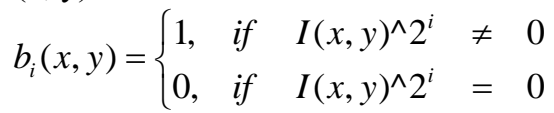

where $b_{i}(x, y)$ is the $i$-th bit-plane and $\mathrm{i}=0,1,2, \ldots \ldots, \log _{2} \mathrm{~N}$ -1 \}. Here $\wedge$ is the binary AND operator. Thus, the grayscale image is a fusion of a number of bit-planes, weighted by their respective grayness.

\section{B. Information Gain Computation}

Let there be two attributes $\mathrm{X}$ and $\mathrm{Y}$. The information gain, denoted by $I(Y ; X)$, of $\mathrm{X}$ with respect to $\mathrm{Y}$ is defined as the reduction in uncertainty about the value of $Y$ when the value of $\mathrm{X}$ is known. The entropy $H(Y)$ measures the uncertainty about the value of $\mathrm{Y}$. When $\mathrm{Y}$ is a discrete variable that assumes the values in $\mathrm{y}_{1}, \mathrm{y}_{2}, \ldots$, $\mathrm{y}_{\mathrm{n}}$, then the entropy of $\mathrm{Y}$ is given by: 


$$
H(Y)=-\sum_{i=1}^{n} P\left(Y=y_{i}\right) \log _{2} P\left(Y=y_{i}\right)
$$

The information gain of $\mathrm{X}$ with respect to $\mathrm{Y}$ may be represented as:

$$
I(Y ; X)=H(X)+H(Y)-H(X, Y)
$$

The joint entropy of $X$ and $Y$ is denoted by $H(X, Y)$ and is defined as

$$
H(X, Y)=-\sum_{i=1}^{k} \sum_{j=1}^{l} P\left(X=x_{i}, Y=y_{j}\right) \log _{2} P\left(X=x_{i}, Y=y_{j}\right)
$$

In this paper, the corner detection is considered to be a binary classification problem. Let us denote the original image by $\mathrm{H}$ and i-th bit-plane of the image by $\mathrm{H}_{\mathrm{i}}$. A random variable $\mathrm{Y}$ is defined as follows.

$$
Y= \begin{cases}1, & \text { if a pixel }(\mathrm{x}, \mathrm{y}) \text { is classified as corner } \\ 0, & \text { if }(\mathrm{x}, \mathrm{y}) \text { is not a corner pixel }\end{cases}
$$

A random variable $\mathrm{X}_{\mathrm{i}}$ is defined for each bit-plane $\mathrm{i},(\mathrm{i}=1,2$, $3, \ldots \ldots \ldots, 8)$, of the image $\mathrm{H}$ as follows:

$X_{i}=\left\{\begin{array}{rr}1, & \text { if a pixel }(\mathrm{x}, \mathrm{y}) \text { is a corner in } \mathrm{i}-\text { th bitplane } \\ 0, & \text { if }(\mathrm{x}, \mathrm{y}) \text { is not a corner in } \mathrm{i}-\text { th bitplane }\end{array}\right.$

Initially, the corners in the original image $\mathrm{H}$ are labelled. Then the expected information gain $\mathrm{I}\left(\mathrm{Y} ; \mathrm{X}_{\mathrm{i}}\right)$ is computed in each of the bitplane $\mathrm{i}$, where $\mathrm{i}=1,2,3, \ldots \ldots \ldots ., 8\}$ in $\mathrm{H}$ by

$$
I\left(Y ; X_{i}\right)=H\left(X_{i}\right)+H(Y)-H\left(X_{i}, Y\right)
$$

\section{PROPOSED ALGORITHM}

In the literature, there exist a number of corner detectors that would give multiple responses in a small neighborhood of the actual corner. This is basically due to the inherent digital nature of the image. Since corners in an image represent those image pixels with high information content, they correspond to higher bit-planes. Therefore, the pixels belonging to the lower bit-planes only can be treated as noise. This is the underlying philosophy of our approach. After fission of a grayscale image into eight bit-planes (binary images), a specific gray-level corner detector on all the bit-planes is used simultaneously. The fission/fusion technique can be used in conjunction with any corner detection algorithm in order to reduce the number of erroneous corners.

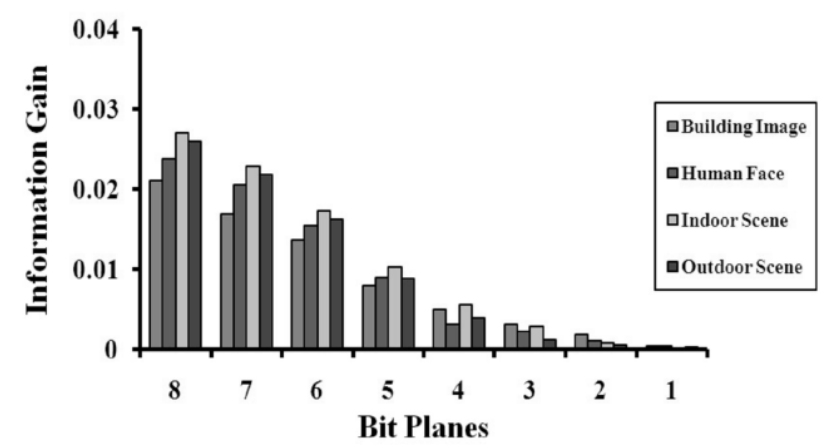

Fig. 1 Mean information gain in individual bit-planes for different types of test images used for training

After obtaining corners from all bit-planes, the information gain is calculated in individual bit-plane $\mathrm{H}_{\mathrm{i}}$. Then information gain in individual bit-plane for all test images is plotted in Fig. 2, and it is observed that information gain is the maximum at the highest $\left(8^{\text {th }}\right)$ bit-plane, which gradually decreases as we move towards lower bit-planes (shown in Fig. 1). In fact, in the lower bit-planes, the information gain is almost negligible. Then starting with the bit-plane with the highest information gain, and gradually the bit-plane with next highest information gain is fused with it and so on and check the number of missed corners after each fusion. For the experimentation purpose, the number of missed corners is plotted with respect to different bit-plane combinations and it is noted that after a certain bit-plane we are not gaining with respect to the number of missed corners. This signifies that after the fusion of bit-planes from the highest to a particular bit-plane, the number of missed corners does not reduce.

For training purpose, more than 200 images of different types have been used, most of them obtained from CMU image a1 [38](see Table 1). During the training phase, different thresholds for different types of images is obtained for which our extension/improvement performed well. It is found from Table 1 that for all categories of images, the average threshold obtained is the sixth $\left(6^{\text {th }}\right)$ bitplane. That particular bit-plane is considered as the thresholded bit-plane and all the lower bit-planes are ignored.

Table 1: Training data (Average threshold for different types of images)

\begin{tabular}{|c|c|c|}
\hline Image types & $\begin{array}{c}\text { Number of images } \\
\text { considered }\end{array}$ & $\begin{array}{c}\text { Average thresholded } \\
\text { bitplane }\end{array}$ \\
\hline Indoor Scene & 74 & 6.22 (approx 6) \\
\hline Outdoor Scene & 58 & 6.03 (approx 6) \\
\hline Human Face & 36 & 5.83 (approx 6) \\
\hline House Image & 52 & 6.23 (approx 6) \\
\hline
\end{tabular}

The detailed algorithm is given below:

1. Decompose an input gray-level image into several bit-planes (binary images each weighted by grayness).

2. Perform corner detection in each bit-plane simultaneously to obtain corners in all of the bit-planes.

3. Compute the information gain from individual bit-planes and observe that the gain gradually decreases as we traverse from higher towards lower bit-plane.

4. Count the number of missed corners identified after fusing the highest bit-plane with a bit-plane with next highest information gain and find that after a particular bit-plane, the fusion of lower bit-planes is not detecting any extra corners, i.e., after a certain bit-plane, we are not gaining in terms of missed corners.

5. Threshold at this point so as to ignore all the lower bit-planes.

6. Perform a fusion of all the higher bit-plane corners.

7. Corners surviving after non-maximum suppression form the resultant set of corners.

\section{EXPERIMENTAL RESULTS}

In order to validate the efficiency of the proposed approach, the improvement of the proposed extension is shown to five popular corner detection methods, namely those proposed by Moravec (hereafter Mor77), Kitchen and Rosenfeld (KR82), Harris and Stephens (HS88), Laganiere (Lag98) and Alkaabi and Deravi (AD04) using image fission/fusion approach. 
The Moravec detector is selected for its historical importance and the remaining four performed well on different types of images. For testing, a large number of images are used, out of which a set of six test images (Fig. 2) are given in the paper as a representative test images. In each of the test images we have assumed the sixth bitplane as the thresholded bitplane. In figure Fig. 3, the mean of different performance measures is plotted mentioned in section 2.2 over the set of test images. Experimentation was done on a desktop computer with Windows 7 operating system, Pentium 4 processor with $3.2 \mathrm{GHz}$ clock speed and 4GB RAM. (a) Building Image

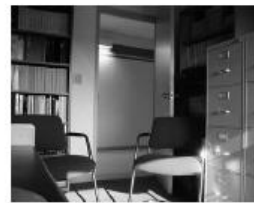

(d) Indoor Scene

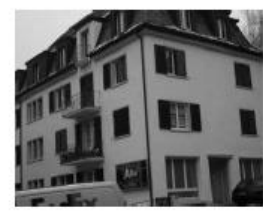

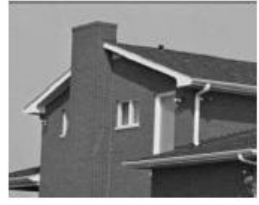

(b) House Image

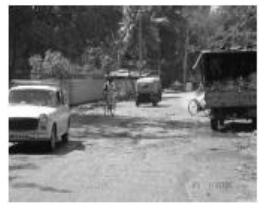

(e) Outdoor Scene-1

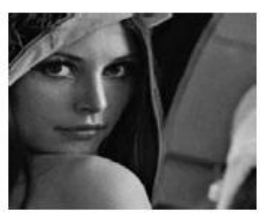

(c) Human Face

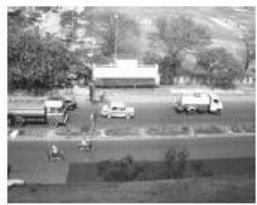

(f) Outdoor Scene-2
Fig. 2. Representative test images used to assess the performance of corner detection algorithms

From the Fig. 3, it is observed that the average value of the detection gradient is always less for the fission/fusion extension of corner detectors over the set of six test images when we compare it with the original detectors. Similar is the fact for the three other performance measures - accuracy measure, detection rate and error rate. Thus it is clear from the results depicted in figures Fig. 3 that proposed extension to each of these five algorithms using fission/fusion performs better compared to the original corner detectors with respect to detection of true corners, false positive (extra) corners and false negative (missed) corners.

In order to establish the efficiency of the proposed improvement, Gaussian noise has been added to the test images (artificially) and the images are rotated by different angles. These distorted images are then used for experiment to compare with other corner detectors. For comparison the performance measures, namely detection rate (DR) and error rate (ER) have been used. Moreover, the average computational time of the proposed algorithm has been calculated and is compared it with other corner detectors stated before. In addition, two more contemporary corner detectors have been considered, proposed by Rosten and Drummond (RD06) and Pedrosa and Barcelos (PB10). The results of the experiment are shown in Fig. 4. It is seen from Fig. 5 that though the proposed algorithm took slightly higher computational time compared to AD04 and RD06 out of seven detectors under comparison, but it performs better than all the detectors with respect to its robustness to noise and repeatability under the transformation rotation.

Moreover, the values of different performance measures with respect to different bitplane combinations for all test images have been plotted (see Fig. 2) in Fig. 6. It is verified from Fig. 6 that if we have changed the bitplane combinations, the results of corner detection algorithm are degraded. This is because the number of false negative (missed) corners remains unchanged, but the number of false positive (extra) corners increases with the increase in bitplanes in the fusion process. It established the fact that in the proposed algorithm, we used the optimum combination of bitplanes for different types of test images.

The threshold is obtained by calculating the information gain across different bitplanes and observing the number of missed corners after fusion higher bit-planes in sequence. This approach is fast because it is operated on binary images instead of graylevel images. First with the help of a training process with the help of different types of images the thresholded bitplane is obtained. Then testing is performed with the threshold obtained from the training phase on various kinds of test images - indoor, outdoor, human face, building images etc. The mean of different performance measures is plotted for different test images.

\section{CONCLUSIONS}

Corner detection from digital images is crucial in various tasks in computer vision. In this paper, a novel fission/fusion based approach to improve the performance of the original corner detection algorithms is presented. Here the corner detection is considered as binary classification problem. Since a graylevel image is first decomposed into (eight) bitplanes, each of which is a binary image, an existing corner-detection algorithm can be applied to all bitplanes simultaneously to extract corners from the bit-planes and finally the higher bitplanes up to some thresholded limit are recombined, with extracted corners followed by non-maxima suppression giving the final set of corners. Results using a representative subset of five corner detectors show that our approach improves the existing corner detection algorithms significantly. Moreover, since for most of the cases the fusion of lower bitplanes is ignored to obtain the final set of corners, the effect of noise components on corner detection is automatically eliminated. This fission/fusion process for corner detection is applicable to other low-level image processing activity such as segmentation, pre-processing etc.

\section{REFERENCES}

1. A. Dutta, A. Kar and B. N. Chatterji (2008a), 'Corner Detection Algorithms in Digital Images in Last Three Decades', IETE Technical Review, Vol. 25, No. 3, $123-134$.

2. F. Mokhtarian and F. Mohanna (2006), 'Performance Evaluation of Corner Detectors using Consistency and Accuracy Measures', Computer Vision and Image Understanding, Vol. 102, No. 1, 81 - 94

3. M. Awrangjeb (2013), 'A Performance Review of Recent Corner Detectors', in Proceedings of International Conference on Digital Image Computing: Techniques and Applications, Hobart, Australia, 1 -8 .

4. N. Sebe, T. Gevers, S. Dijkstra and J. van de Weijer (2007), 'Evaluation of intensity and color corner detectors for affine invariant salient regions', Proceedings of the Conference on Computer Vision and Pattern Recognition Workshop, Washington, USA.

5. P. Tissainayagam and D. Suter (2004), 'Assessing the Performance of Corner Detectors for Point Feature Tracking Applications', Image and Vision Computing, Vol. 22, No. 8, 663 - 679.

6. Z. Zheng, H. Wang and E. K. Teoh (1999), 'Analysis of Gray Level Corner Detection', Pattern Recognition Letters, Vol. 20, No. 2, pp. $149-162$. 
7. N. Dey, A. B. Roy, P. Das, A. Das and S. S. Chaudhuri (2012a) 'Detection and measurement of arc of lumen calcification from intra vascular ultrasound using Harris corner detection', In NCCCS 2012: IEEE National Conference on Computing and Communication Systems, Durgapur, India, $1-6$.

8. N. Dey, A. B. Roy, P. Das, A. Das and S. S. Chaudhuri (2012b) 'Optical cup to disc ratio measurement for glaucoma diagnosis using Harris corner', in ICCCNT 2012: Proceedings of Third International Conference on Computing Communication \& Networking Technologies, Coimbatore, India, $1-5$.

9. N. Dey, B. Nandi, A. B. Roy, D. Biswas, A. Das and S. S. Chaudhuri (2013), 'Analysis of blood cell smears using stationary wavelet transform \& Harris corner detection', Book Chapter, Recent Advances in Computer Vision and Image Processing, Methodologies and Applications, IGI Global, 357 - 370.

10. A. Dutta, A. Kar and B. N. Chatterji (2008b), 'Comparing and Evaluating Intensity Based Spatial Domain Corner Detectors', International Journal on Information Processing, Vol. 2, No. 4, 48 55.

11. H. P. Moravec (1977), 'Towards Automatic Visual Obstacle Avoidance', Proceedings of 5th International Joint Conference on Artificial Intelligence, Cambridge, MA, 584.

12. L. Kitchen and A. Rosenfeld (1982), 'Gray Level Corner Detection', Pattern Recognition Letters, Vol. 1, No. 2, 95 - 102.

13. C. Harris and M. Stephens (1988), 'A Combined Corner and Edge Detector', Proceedings of 4th Alvey Vision Conference, Manchester, UK, $147-151$

14. S. M. Smith and J. M. Brady (1997), 'SUSAN - A New Approach to Low-Level Image Processing', International Journal of Computer Vision, Vol. 23, No. 1, $45-78$.

15. M. Trajkovic and M. Hedley (1998), 'Fast Corner Detection', Image and Vision Computing, Vol. 16, No. 2, 75 - 87.

16. S. Bae, I. S, Kweon and C. D. Yoo (2002), 'COP: A New Corner Detector', Pattern Recognition Letters, Vol. 23, No. 11, 1349 - 1360.

17. K. Mikolajczyk and C. Schmid (2004), 'Scale and Affine Invariant Interest Point Detectors', International Journal of Computer Vision, Vol. 60, No. 1, 63 - 86 .

18. S. Alkaabi and F. Deravi (2004), 'Candidate Pruning for Fast Corner Detection', Electronic Letters, Vol. 40, No. 1, 18 - 19.

19. E. Rosten and T. Drummond (2006), 'Machine Learning for High-Speed Corner Detection', Proceedings of European Conference on Computer Vision, Graz, Austria, 430 - 443.

20. A. Dutta, A. Kar and B. N. Chatterji (2011), 'Adaptive Window-based Corner Detection Algorithm for Gray-scale Images', IETE Journal of Research, Vol. 57, No. 3, 287 - 294.

21. Z. Y. Zeng, Z. Q. Jiang, Q. Chen and P. F. He (2012), 'An Improved Corner Detection Algorithm Based on Harris', Advanced Engineering Forum, Vols. 6 - 7, $717-721$

22. X. Liu, J. Dai, Y. Jia and R. Liu (2014), 'Caption Region Detection in Video Images by Improved Corner Detector', International Journal of Signal Processing, Image Processing and Pattern Recognition, Vol. 7, No. 4, $409-420$

23. A. Dutta (2015), 'Local Information Based Approach to Corner Detection', International Journal of Application or Innovation in Engineering \& Management, Vol. 4, No. 1, 186 - 190.
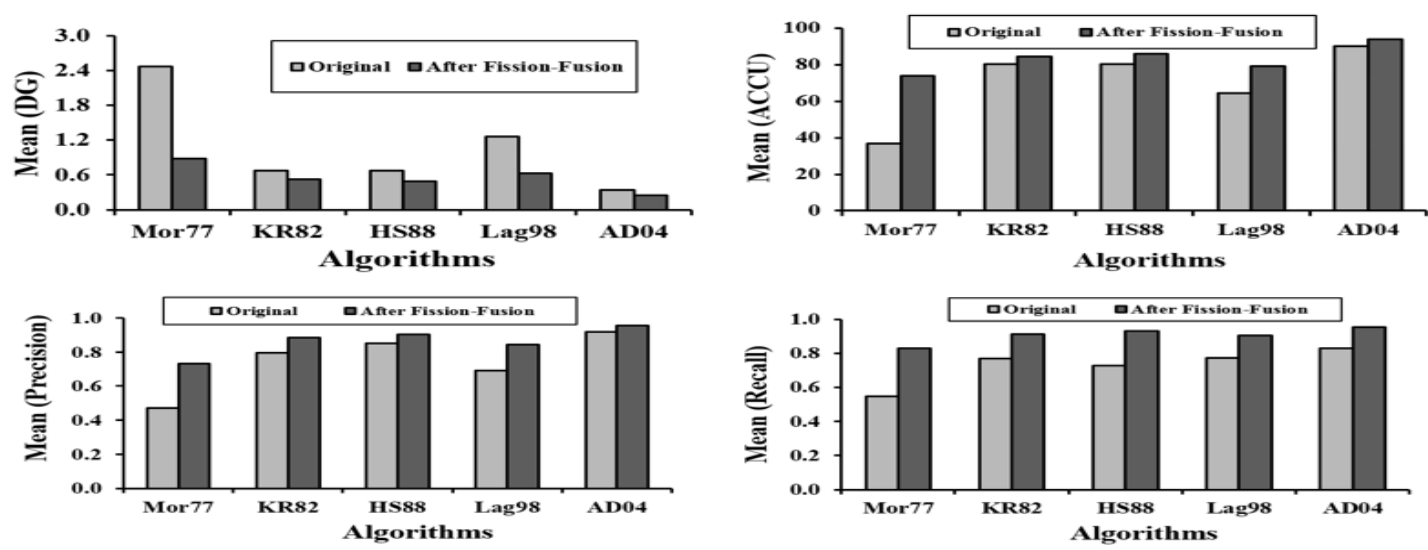

Fig. 3. Improvement of bit-plane extension with respect to different performance measures 
An Improvement to a Class of Intensity based Spatial Domain Corner Detection Algorithms using Image Fission and Fusion

Comparison with respect to robustness to noise and rotation of images

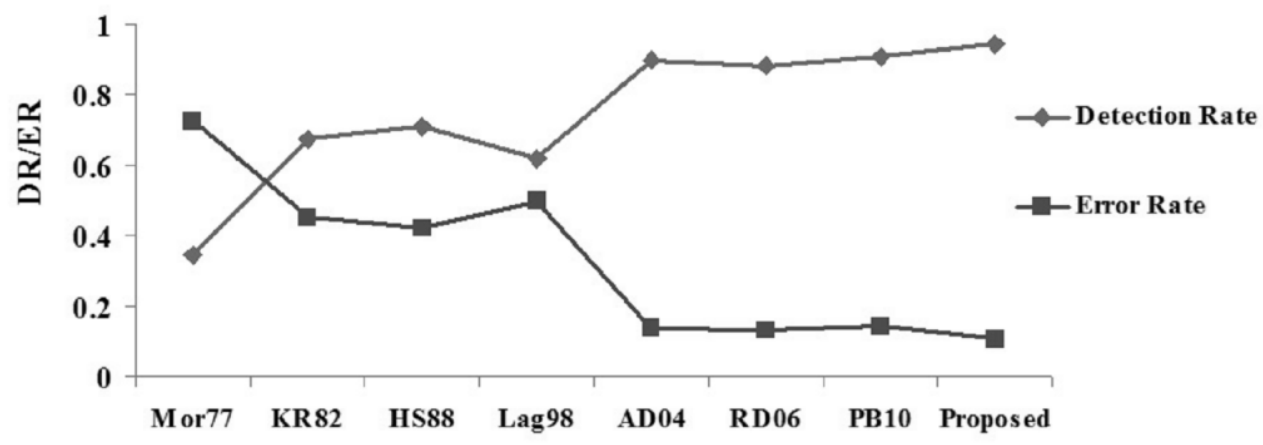

Algorithms

Fig. 4. Improvement of the proposed algorithm with respect to different transformations and noise

\section{Comparison with respect to Computational Time}

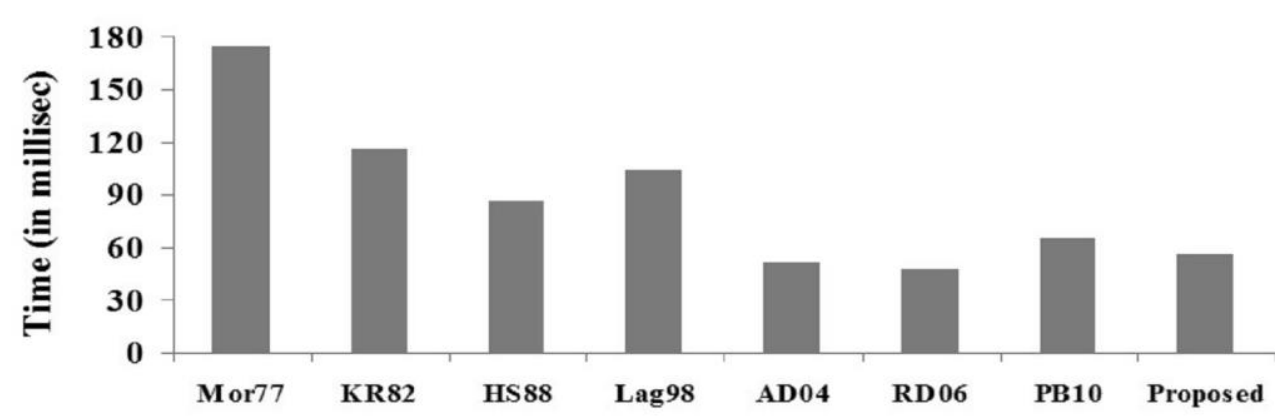

Fig. 5. Improvement of the proposed algorithm with respect to computational time
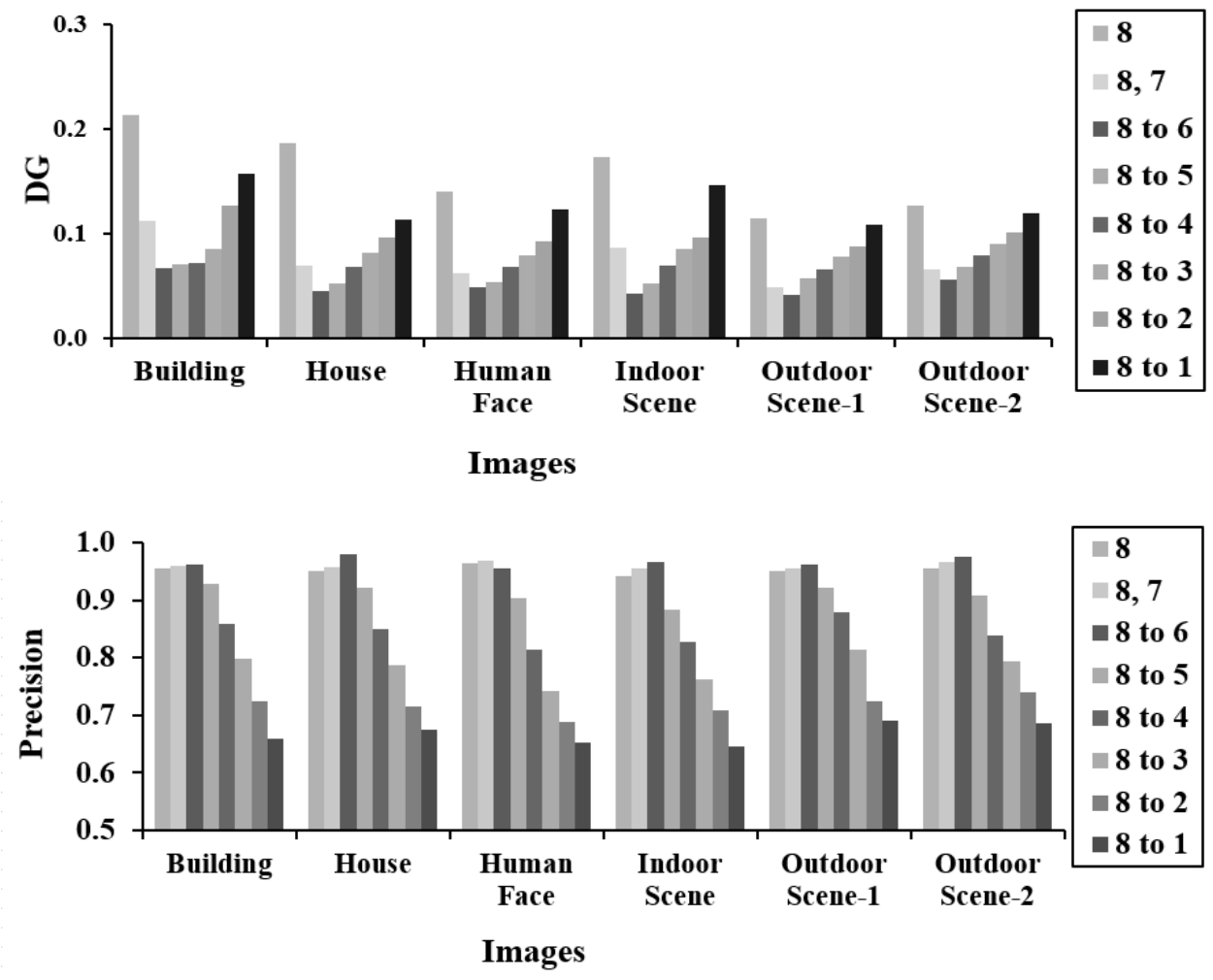


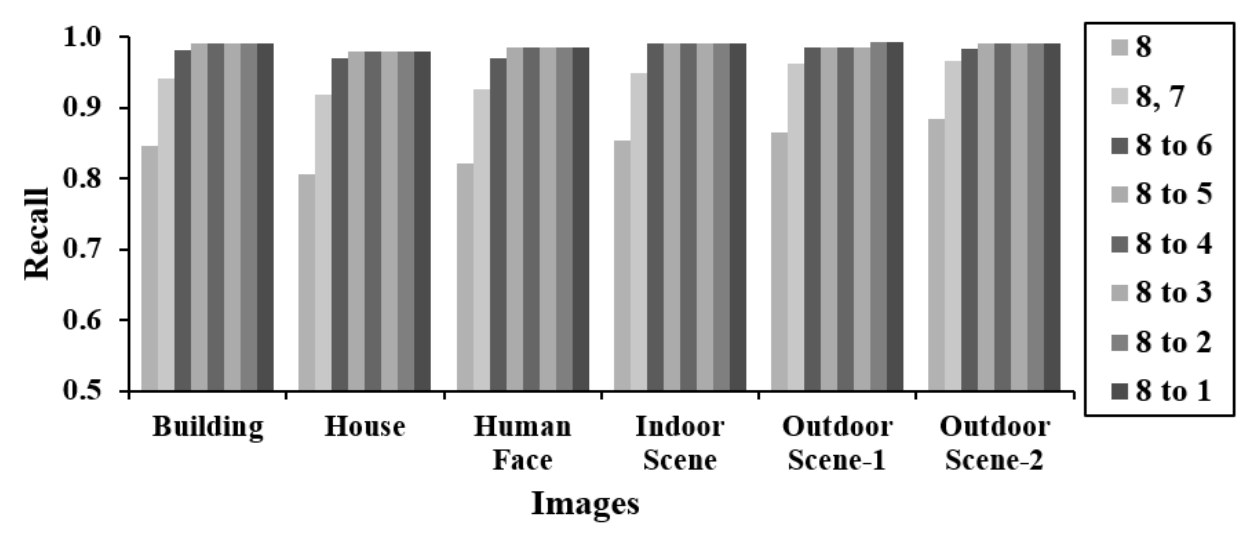

Fig. 6. Values of different performance measures with different bit-plane combinations

\section{AUTHORS PROFILE}

Dr. Ambar Dutta did his B.Sc. in Mathematics Honours from Presidency College, Kolkata, Masters and $\mathrm{PhD}$ in Computer Science and Engineering from Jadavpur University, Kolkata. After serving Birla Institute of Technology, Mesra for more than 14 years, he is at present working as Associate Professor at Amity Institute of Information Technology, Amity University, Kolkata. He published more than 45 quality papers in reputed journals and conferences. His research interests include image and video processing, machine learning, information retrieval, predictive data analytics etc. He is an active reviewer of different reputed international journals. He is a senior life member of different professional bodies like Computer Society of India, ACM, IACSIT etc. 